\title{
Correlation between BRCA1 expression and clinicopathological factors including brain metastases in patients with non-small-cell lung cancer
}

\author{
Mariam Gachechiladze ${ }^{\mathrm{a}}$, Ivo Uberall ${ }^{\mathrm{a}, \mathrm{b}}$, Vitezslav Kolekc, Jiri Klein ${ }^{\mathrm{d}}$, Veronika Krejcia,b, Jitka Stastna ${ }^{\mathrm{a}}$, Lenka Radova ${ }^{\mathrm{e}}$, \\ Eddie Fridman ${ }^{\mathrm{f}}$ Josef Skarda ${ }^{\mathrm{a}, \mathrm{b}}$
}

Background. Previously identified as a breast and ovarian cancer susceptibility gene, BRCA1 has gained major scientific interest as a potential prognostic and/or predictive marker for various tumors, including non-small-cell lung cancer (NSCLC), the leading cause of cancer related mortality worldwide. BRCA1 plays a central role in DNA damage response (DDR. It undergoes phosphorylation by various DDR kinases at different serine residues, of which ser 1524 is known to be specifically phosphorylated by ATM in response to genotoxic stress.

Methods. We performed BRCA1 immunohistochemistry on several tissue microarrays (TMAs) of 113 early (I, II stage) and advanced (III, IV stage) NSCLCs, using MS1 10 antibody against the BRCA1 N-terminal and S1524 antibody against the phosphorylated form of BRCA1 protein at ser1524 (Abcam). Patients with III and IV stage disease were treated by adjuvant cisplatin-based chemotherapy. Staining results were correlated with overall survival (OS), disease free survival (DFS) and with the occurrence of brain metastases.

Results. BRCA1 S1524 nuclear positivity was significantly correlated with longer OS and DFS in stage I and II patients $(P<0.05)$, while OS and DFS were shorter in $\mathrm{S} 1524$ positive stage III and IV patients $(P<0.05)$. No significant correlation was found with brain metastases.

Conclusion. The results show that BRCA1 phosphorylaton, at least in ser1524, differentiates the fate of early and advanced NSCLC as well as response to chemotherapy, but the underlying mechanisms are not completely understood. Detection of phosphorylated forms of BRCA1 might serve as a useful prognostic and predictive marker for patients with NSCLC.

Key words: BRCA1, brain metastases, non-small-cell lung cancer

Received: January 3, 2012; Accepted with revision: October 19, 2012; Available online: November 1, 2012 http://dx.doi.org/10.5507/bp.2012.099

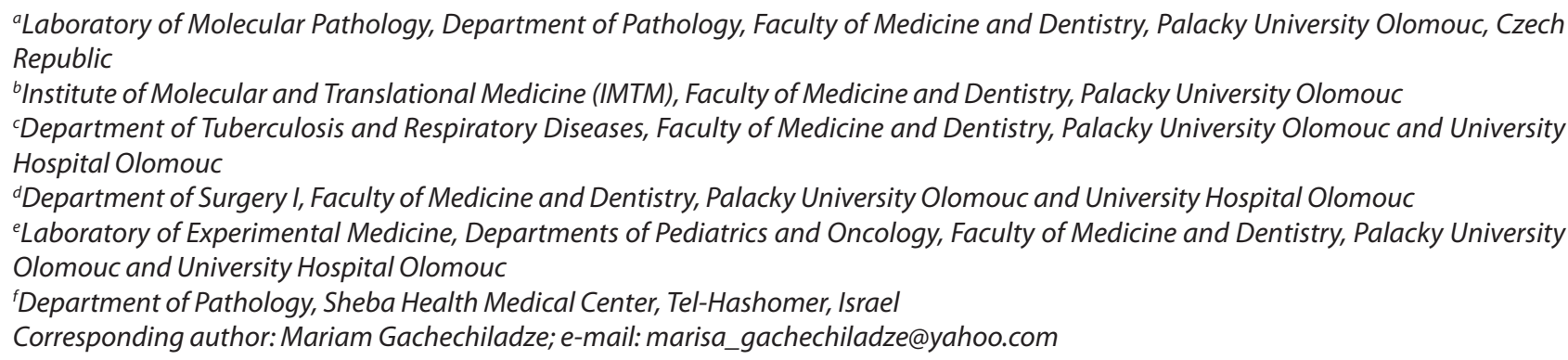

\section{INTRODUCTION}

Non-small-cell lung cancer (NSCLC) is the leading cause of cancer related mortality and one of the major public health problems in the world. More than $75 \%$ of patients are diagnosed at advanced/metastatic stages of disease with a median survival time of only 10-12 month ${ }^{1,2}$. Despite recent improvements in treatment modalities, intrinsic or acquired drug resistance still represents the major impediment to successful treatment.

The main prognostic factors used in routine clinical practice for patients with operable NSCLC are pulmonary symptoms, tumor size, histological type, metastases to lymph nodes and vascular invasion ${ }^{3}$. For patients with advanced and/or inoperable NSCLC, the most predictive indicators of survival after systemic chemotherapy are disease stage, performance status, and weight loss of more than $10 \%\left(\right.$ ref. $\left.^{4}\right)$. However, these factors are not sufficient for precise evaluation of individual patient prognosis and for predicting response after a given chemotherapy. An essential step to reducing the mortality from NSCLC is understanding the molecular basis of its progression, metastases and drug resistance.

BRCA1 (breast cancer 1) is a $100 \mathrm{~kb}, 220 \mathrm{kD}$, multifunctional nuclear phosphoprotein, ubiquitously expressed in all tissues and serves as a tumor suppressor in part, as a "caretaker", as well as "gatekeeper" in preserving genomic stability 5 . It has been implicated in normal cellular functions including cell cycle regulation, replication, mitotic spindle assembly, transcription regulation and higher chromatin hierarchical control ${ }^{5}$. Also BRCA1 plays a central role in DNA damage response (DDR) through direct interaction with DDR proteins, as well as by regulation of gene transcription ${ }^{6}$. 
Germline mutations of BRCA1 have been associated with increased risk of breast, ovarian, and more recently, prostate cancer ${ }^{6-8}$. Breast cancers arising in patients with BRCA1 mutations are usually high grade, poorly differentiated tumors, characteristically rapid progression without an in situ component, higher incidence of metastasis and poor prognosis. The expression of BRCA1 is often reduced in sporadic breast and ovarian cancers ${ }^{5}$ and these tumors share some similarities with hereditary, BRCA1 mutated tumors. It is also known that increased BRCA1 protein expression in prostate cancer is associated with significantly higher Gleason score, and more advanced stage $^{6}$, as well as a poor outcome in thymic epithelial tumors ${ }^{9}$. A wealth of data from cell lines, mouse models and more recently from clinical studies have shown that BRCA1 can modulate response to chemotherapy ${ }^{10-13}$.

BRCA1 mutations are not found in lung cancer, but decreased BRCA1 mRNA and protein expression due to promoter hypermethylation were found in $30 \%$ of NSCLC patients mostly in adenocarcinoma subtypes $(P<0.014)$ $\left(\right.$ ref. $\left.{ }^{14}\right)$. Several studies have examined the correlation of BRCA1 mRNA expression with response to chemotherapy in clinical settings. Overexpression of BRCA1 mRNA was strongly associated with poor survival in NSCLC patients, after cisplatin based chemotherapy ${ }^{15-18}$ but these findings were not confirmed by immunohistochemistry ${ }^{19-21}$.

We set out to study the immunohistochemical expression of BRCA1 protein in NSCLC and assess associations with clinicopathological factors, including overall survival (OS), disease free survival (DFS) and the occurrence of brain metastases.

\section{MATERIALS AND METHODS}

\section{Patients and samples}

Archival formalin-fixed, paraffin-embedded tissue samples of non-small-cell carcinomas of the lung derived from patients operated between 1996 and 2000, were obtained. Each sample was diagnosed by two independent pathologists according to the WHO classification. The patient cohort consisted of 88 men and 25 women. Of these, 27 were in stage I, 9 in stage II, 52 patients were in stage III and 10 patients were in stage IV. Brain metastases were present in all (10) stage IV patients. Information about disease stage was not available in 15 cases. 27 patients had received neoadjuvant chemotherapy. Of these, 2 patients had undergone both adjuvant and neoadjuvant chemotherapies. 10 patients had received only adjuvant chemotherapy. Out of 113 patients, 76 did not receive adjuvant or neoadjuvant chemotherapy. The overall survival of patients in months was determined from clinical data. The disease-specific survival was determined as the interval from diagnosis to the disease-specific death. Median and disease-free survival rates are given in Table 1.

\section{Tissue microarray construction}

Tumour tissue microarrays were constructed using 113 formalin-fixed primary lung cancer tissue samples. The tissue area for sampling was based on visual alignment with the corresponding $\mathrm{H} \& \mathrm{E}$ stained section on a slide. Three to five tissue cores (diameter: $2.00 \mathrm{~mm}$; height: 3-4 $\mathrm{mm}$ ) taken from a donor tumour block were placed in a recipient paraffin block with a manual tissue microarrayer (Beecher Instruments, Sun Prairie, WI, USA).

Table 1. Clinicopathological characteristics of NSCLC patients.

\begin{tabular}{|c|c|c|c|c|c|}
\hline & All Patients & I & II & III & IV \\
\hline $\mathrm{N}$ & 113 & 27 & 9 & 52 & 10 \\
\hline Median OS (month) & 46.03 & 93.18 & 40.95 & 44.03 & 13.13 \\
\hline Mean OS (month) & 58.62 & 86.19 & 48.64 & 59.86 & 35.11 \\
\hline Median DFS & 38.82 & 80.69 & 31.81 & 21.71 & 10.57 \\
\hline Mean DFS & 53.70 & 81.03 & 44.90 & 53.21 & 31.40 \\
\hline Age & 60 & 62 & 57 & 61.5 & 54.5 \\
\hline \multicolumn{6}{|l|}{ Gender } \\
\hline Male & 88 & 19 & 8 & 40 & 8 \\
\hline Female & 25 & 8 & 1 & 12 & 2 \\
\hline \multicolumn{6}{|l|}{ Histology } \\
\hline $\mathrm{ADC}$ & 43 & 13 & 4 & 16 & 6 \\
\hline $\mathrm{SCC}$ & 49 & 10 & 3 & 26 & 2 \\
\hline Others & 21 & 4 & 2 & 10 & 2 \\
\hline \multicolumn{6}{|l|}{ Neoadjuvant CHT } \\
\hline CHT & 27 & 2 & 0 & 22 & 3 \\
\hline Observation & 72 & 25 & 9 & 30 & 6 \\
\hline Adjuvant CHT & 10 & 1 & 1 & 6 & 2 \\
\hline $\mathrm{Pt} / \mathrm{NVB}$ & 0 & 0 & 0 & 0 & 0 \\
\hline Pt/TAX & 2 & 0 & 0 & 2 & 0 \\
\hline Other & 8 & 1 & 1 & 4 & 2 \\
\hline
\end{tabular}

NSCLC, non-small-cell lung cancer; ADC, adenocarcinoma; SCC, squamous cell carcinoma; OS, overall survival; DFS, disease free survival; CHT, chemotherapy; NVB, navelbine; TAX, taxol. 
A core of normal tissue was punched from each case, and $4 \mu$ sections of the resulting microarray block were used for immunohistochemical analysis.

\section{Immunohistochemistry}

Formalin-fixed and paraffin-embedded tissue sections ( $4 \mu$ ) of NSCLC were deparaffinized in xylene and rehydrated by washing in serial dilutions of ethanol. BRCA1 heat induced antigen retrieval was performed in an automatic multifunctional microwave tissue processor $(\mathrm{T} / \mathrm{T}$ MEGA) at $95{ }^{\circ} \mathrm{C}$, over $5 \mathrm{~min}$, using citrate buffer at $\mathrm{pH} 6$. Endogenous peroxidase activity was blocked with $0.3 \%$ hydrogen peroxide for $15 \mathrm{~min}$. The monoclonal MS110 (Abcam) antibody against the N-terminal of BRCA1 protein and polyclonal S1524 (Abcam) antibody against the phosphorylated form of BRCA1 at ser 1524 residue were then added (1:150 dilution) and incubated for $1 \mathrm{~h}$ at room temperature. Tissue sections without primary antibody addition served as a negative control. Breast cancer tissue was used as a positive control. Antigen was visualized using the EnVision kit (DAKO A S, Glostrup, Denmark) for $60 \mathrm{~min}$. The slides were then counterstained with haematoxylin and mounted in an aqueous medium.

\section{Analysis of results}

BRCA1 protein expression was evaluated semiquantitatively. Cases with nuclear and/or cytoplasmic BRCA1 reactivity in $>10 \%$ of cancer cells were considered as positive. Analysis of data was performed using the computer software SPSS for Windows (version 10.0). Nonparametric comparison between independent groups was carried out using the Kruskal-Wallis test and $P$-values $<0.05$ were considered statistically significant.

\section{RESULTS}

BRCA1 MS110 staining showed mainly nuclear positivity in $28 \%$ of NSCLC cases. Only 2 cases were positive in both - nucleus and cytoplasm and none of them in only the cytoplasm. BRCA1 S1524 antibody was positive in $27 \%$ of NSCLC cases, from which 11 cases were only nuclear, 13 - n/c and only one case showed cytoplasmic positivity. Exact numbers are given in Table 2.

Statistical analysis of the data showed that only BRCA1 S1524 nuclear positivity was significantly correlated with longer OS and DFS in stage I and II patients $(P<0.05)$ (Fig. 1,2), whilst OS and DFS was shorter in $\mathrm{S} 1524$ positive stage III and IV patients $(P<0.05)$ (Fig. 3,4). No significant correlation was found between BRCA1 MS110 expression and given clinicopathological factors. There was also no significant correlation between either MS110 or S1524 expression and the occurrence of brain metastases.

\section{DISCUSSION}

Earlier reports found no correlation between BRCA1 immunohistochemical expression and NSCLC patient survival or other clinicopathological factors. In all these studies, the MS110 antibody was used for BRCA1 evaluation and our results are consistent with previous reports.

The BRCA1 gene expands the $100 \mathrm{~kb}$ region, produces 1863aa full length protein and several alternative splicing isoforms. The MS110 antibody recognizes the $\mathrm{N}$-terminal 1-304 amino acids of protein. This is the only currently proven antibody for BRCA1 protein study, but these amino acid sequences are also retained in alternative forms of BRCA1. This might be one reason for the discrepancy between mRNA and protein study results in lung cancer patients.

On the other hand mRNA expression alone does not reflect the presence of the functional protein. From a practical point of view, immunohistochemical detection BRCA1 protein is a more reliable and useful tool for pre-

Table 2. BRCA1 expression in NSCLC patients.

\begin{tabular}{|c|c|c|c|c|c|}
\hline & & \multicolumn{2}{|c|}{ BRCA1 MS110 n/c } & \multicolumn{2}{|c|}{ BRCA1 S1524 n/c } \\
\hline & & $\begin{array}{c}\text { Positive } \\
>10 \%\end{array}$ & $\begin{array}{c}\text { Negative } \\
<10 \%\end{array}$ & $\begin{array}{c}\text { Positive } \\
>10 \%\end{array}$ & $\begin{array}{c}\text { Negative } \\
<10 \%\end{array}$ \\
\hline \multicolumn{6}{|l|}{ Stage } \\
\hline & All Patients & $25 / 1$ & $65 / 89$ & 24 & 66 \\
\hline & I & $6 / 0$ & $15 / 21$ & $9 / 5$ & $16 / 20$ \\
\hline & II & $3 / 0$ & $6 / 9$ & $0 / 1$ & $7 / 1$ \\
\hline & III & $14 / 1$ & $37 / 50$ & $11 / 7$ & $37 / 11$ \\
\hline & IV & $2 / 0$ & $7 / 9$ & $4 / 1$ & $6 / 9$ \\
\hline \multicolumn{6}{|c|}{ Histology } \\
\hline & All Patients & $28 / 1$ & $76 / 103$ & $25 / 18$ & $79 / 86$ \\
\hline & Spinocellular & $13 / 1$ & $33 / 40$ & $14 / 17$ & $25 / 32$ \\
\hline & Adenocarcinoma & $7 / 0$ & $43 / 46$ & $8 / 8$ & $39 / 39$ \\
\hline & Others & $8 / 0$ & $9 / 17$ & $3 / 3$ & $15 / 15$ \\
\hline \multicolumn{6}{|c|}{ Chemotherapy } \\
\hline & Neoadjuvant & $24 / 0$ & $20 / 24$ & $7 / 1$ & $19 / 25$ \\
\hline & Adjuvant & $9 / 0$ & $7 / 9$ & $2 / 3$ & $8 / 7$ \\
\hline & Both & $0 / 0$ & $0 / 0$ & $0 / 0$ & $1 / 1$ \\
\hline
\end{tabular}



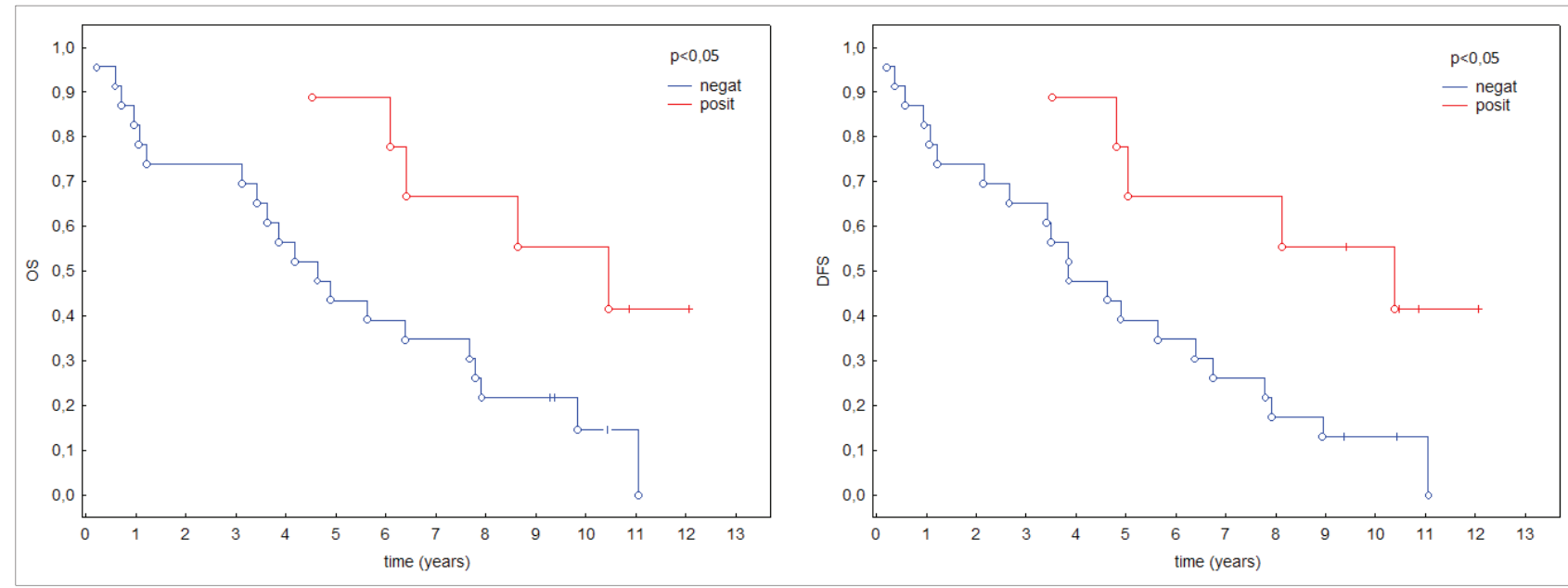

Fig. 1. Correlation of BRCA1 S1524 nuclear expression with OS and DFS in patients with I-II stage NSCLC.

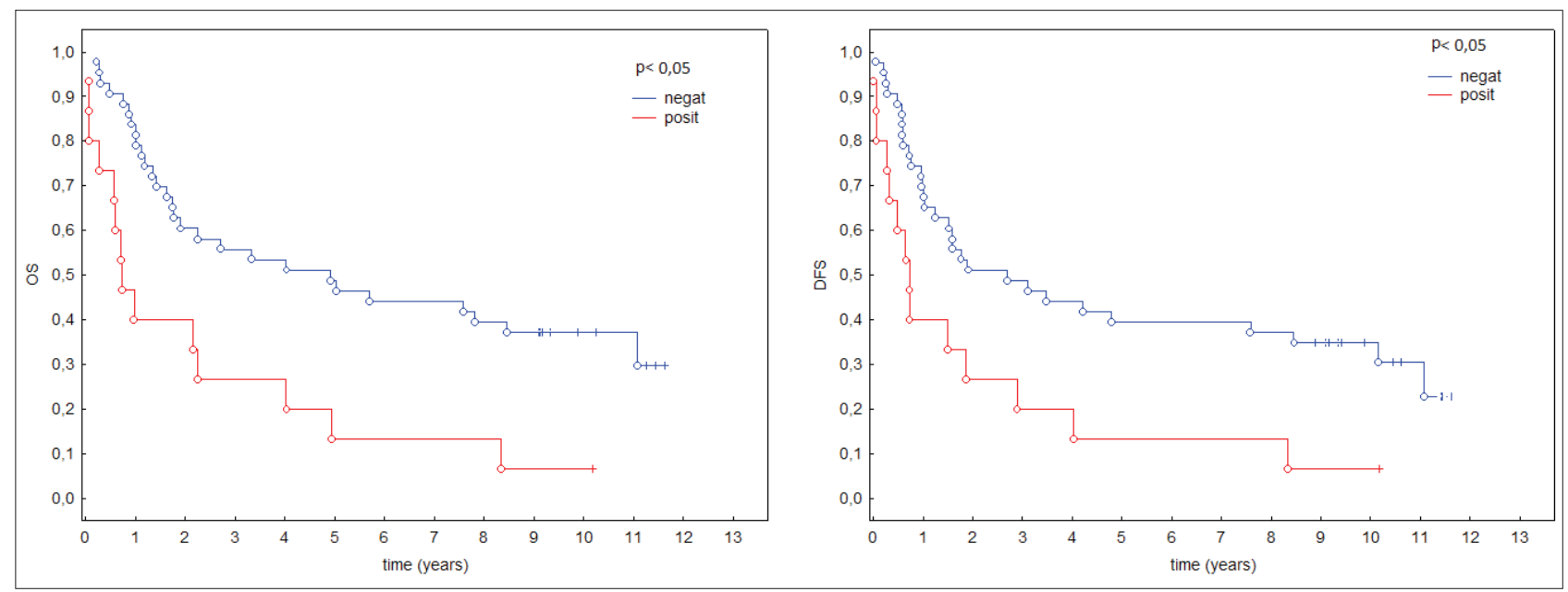

Fig. 2. Correlation of BRCA1 S1524 nuclear expression with OS and DFS in patients with III-IV stage NSCLC.

cise evaluation of patient prognosis. To the best of our knowledge, no one has yet examined BRCA1 phospo-1524 expression in NCSLC patients. BRCA1 phosphorylation is an important means by which its cellular functions are regulated. BRCA1 undergoes specific phosphorylation by cell cycle checkpoint kinases in different serine residues, of which ser 1524 is known to be specifically phosphorylated by ATM after radiation induced DNA damage ${ }^{23}$. Due to its various functions in DNA reparation, including nucleotide excision repair (NER) and double-strand break (DSB) repair, BRCA1 is actively investigated as a resistance marker for cytotoxic chemotherapeutic drugs in lung cancer patients ${ }^{24-27}$.

In our preliminary study, we evaluated phospho-BRCA1 expression in early stage, operable NSCLC cases. It is known that tumor expression of two other DNA-repair genes, RRM1 and ERCC1, signify a survival advantage for patients with early-stage NSCLC ( ref. $^{28}$ ) given only surgical treatment. In our study, phospho-BRCA1 expression was also significantly correlated with longer OS and DFS in stage I and II NSCLC patients.
In advanced stage NSCLC, phospho-BRCA1 expression is associated with shorter OS and DFS and our results are consistent with previous reports showing that DNA repair proteins and particularly BRCA1 is a modulator of chemotherapy in advanced NSCLC. We found no significant difference between treatment groups.

As mentioned above, BRCA1 associated breast and ovarian tumors are distinguished as having high metastatic potential. One study found significantly higher number of brain metastases in BRCA1 dependent breast cancer patients ${ }^{29}$. In our study we had only ten patients with brain metastases and no correlation was found with BRCA1 expression, but sample size is too small to draw any conclusions.

Our study was preliminary, included small sample size and there was no uniform protocol for patient treatment. Also we did not study correlation between BRCA1 and other DNA repair proteins. Hence we are not able to draw sharp conclusions, but we think that further studies of BRCA1 protein expression in larger patient cohort with more defined treatment modalities is worth to conduct. 

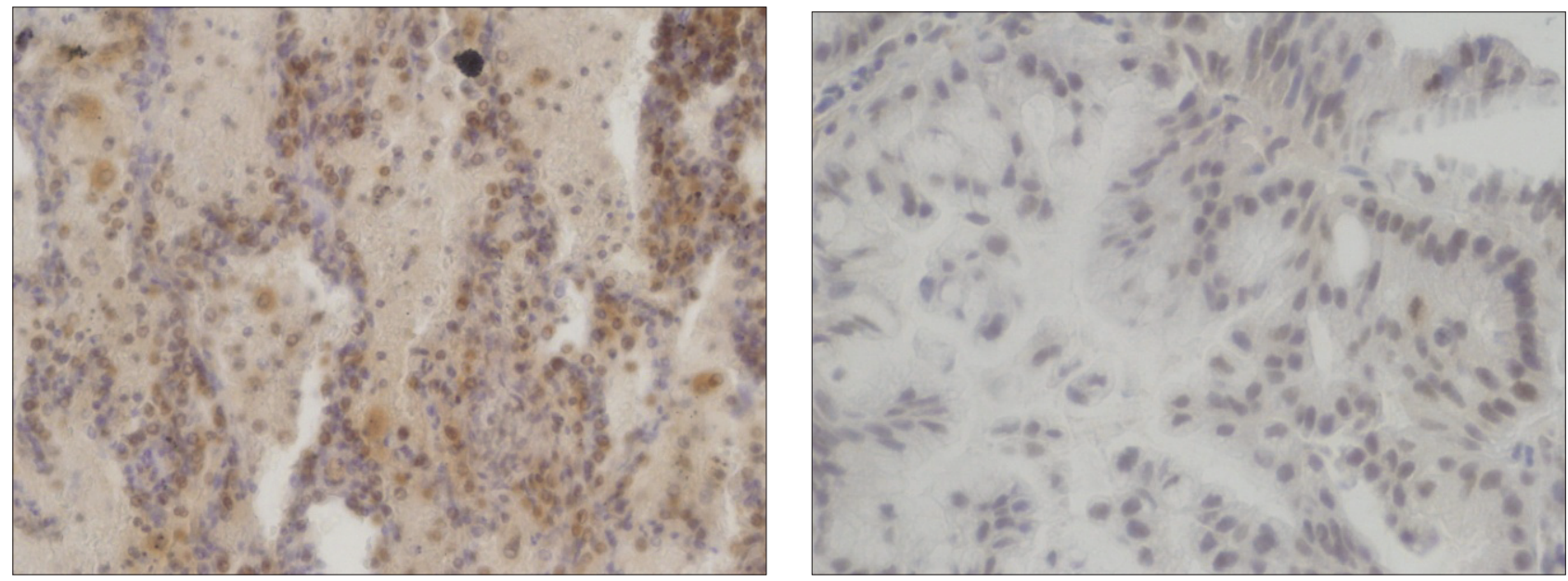

Fig. 3. BRCA1 MMS110 positivity, IHC, x200.
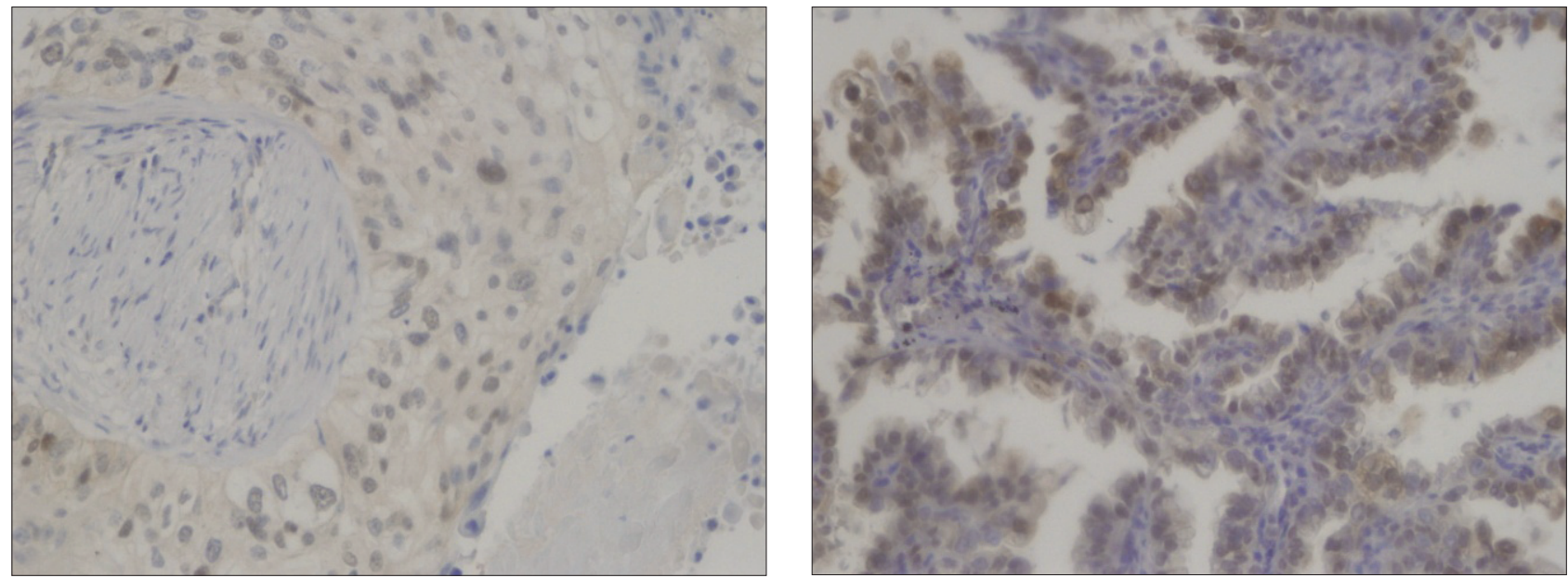

Fig. 4. BRCA1 S1524 positivity, IHC, x200.

\section{CONCLUSIONS}

The results suggest that phospho-BRCA1 immunohistochemical expression might serve as a useful prognostic marker for early stage, operable NSCLC and further strengthens the idea that the discrepancy between mRNA and protein studies in advanced NSCLC patients, may be due to the nature of the BRCA1 protein and inability to detect the full length, functional protein. Antibodies against the phosphorylated form of BRCA1 might be a useful tool to solve this problem.

\section{ACKNOWLEDGEMENTS}

This publication was supported by a grant MSM 6198959216, IGA MZCR NT/13569, IGA MZCR NS/9959-3, IGA MZ 10285-3 200, NS/10259 and project Biomedicine for regional development and human resources (BIOMEDREG) C.1.05/2.1.00/01.0030. M. Gachechiladze was also supported by GACR 303/09/ HO48 from the Grant Agency of the Czech Republic.

\section{REFFERENCES}

1. Jemal A, Siegel R, Ward E, Hao YP, Xu JQ, Murray T, Thun M. Cancer statistics. CA Cancer Journal for Clinicians 2008;58:71-96.

2. Delbaldo C, Michiels S, Rolland E, Syz N, Soria JC, Le Chevalier T, Pignon JP. Second or third additional chemotherapy drug for nonsmall cell lung cancer in patients with advanced disease. Cochrane Database Syst Rev 2007;(4):CD004569.

3. National Cancer Institute, General Information About Non-Small Cell Lung Cancer. 2010

4. Brundage MD, Davies D, Mackillop WJ. Prognostic factors in nonsmall cell lung cancer: a decade of progress. Chest 2002;122:103757.

5. Rosen EM, Fan S, Isaacs C. BRCA1 in hormonal carcinogenesis: basic and clinical research. Endocrine Related Cancer 2005;12(3):533-48.

6. Yarden RI, Papa MZ. BRCA1 at the crossroad of multiple cellular pathways: approaches for therapeutic interventions. Molecular Cancer Therapy 2006;5(6):1396-1404.

7. Agalliu I, Gern R, Leanza S, Burk RD. Associations of high-grade prostate cancer with BRCA1 and BRCA2 founder mutations. Clinical Cancer Research 2009;15(3):1112-20.

8. Fiorentino $M$, Judson G, Penney K, Flavin R, Stark J, Fiore Ch, Fall K, Martin N, Ma J, Sinnott J, Giovannucci E, Stampfer M, Sesso HD, Kantoff PW, Finn S, Loda M, Mucci L. Immunohistochemical expression of BRCA1 and lethal prostate cancer. Cancer Research 2010;70(8):3136-9. doi:10.1158/0008-5472.CAN-09-4100.

9. Kaira K, Serizawa M, Koh Y, Miura S, Kaira R, Abe M, Nakagawa K, Ohde Y, Okumura T, Naito T, Murakami H, Takahashi T, Kondo H, 
Nakajima T, Endo M, Yamamoto N., Expression of excision repair cross-complementation group 1, breast cancer susceptibility 1, and $\beta$ III-tubulin in thymic epithelial tumors. Journal of Thorac Oncology 2011;6(3):606-13.

10. Lafarge S, Sylvain V, Ferrara M, Bignon YJ. Inhibition of BRCA1 leads to increased chemoresistance to microtubule-interfering agents, an effect that involves the JNK pathway. Oncogene 2001;20:6597-606.

11. Husain A, He G, Venkatraman ES, Spriggs DR.BRCA1 up-regulation is associated with repair-mediated resistance to cisdiamminedichloroplatinum(II). Cancer Research 1998;58:1120-3.

12. Bhattacharyya A, Ear US, Koller BH, Weichselbaum RR, Bishop DK. The breast cancer susceptibility gene BRCA1 is required for subnuclear assembly of Rad51 and survival following treatment with the DNA cross-linking agent cisplatin. Journal Biological Chemistry 2000;275:23899-903.

13. Stordal B, Davey R. A systematic review of genes involved in the inverse resistance relationship between cisplatin and paclitaxel chemotherapy: role of BRCA1. Current Cancer Drug Targets 2009;9:354 65.

14. Marsit CJ,Liu M,Nelson HH,Posner M,Suzuki M Kelsey KT. Inactivation of the Fanconi anemia/BRCA pathway in lung and oral cancers: implications for treatment and survival. Oncogene 2004;23:1000-4.

15. Rosell R, Cuello M, Cecere F, Santarpia M, Reguart N, Felip E, Taron M. Usefulness of predictive tests for cancer treatment. Bull Cancer 2006;93:E101-8.

16. Taron M, Rosell R, Felip E, Mendez P, Souglakos J, Ronco MS, Queralt C, Majo J, Sanchez JM, Sanchez JJ, Maestre J. BRCA1 mRNA expression levels as an indicator of chemoresistance in lung cancer. Human Molecular Genetics 2004;13(20):2443-9.

17. Boukovinas I, Papadaki C, Mendez P, Taron M, Mavroudis D, Koutsopoulos A, Sanchez-Ronco M, Sanchez JJ, Trypaki M, Staphopoulos E, Georgoulias V, Rosell R, Souglakos J Tumor BRCA1, RRM1 and RRM2 mRNA Expression Levels and Clinical Response to First-Line Gemcitabine plus Docetaxel in Non-Small-Cell Lung Cancer Patients. PLoS ONE2008;3:11-15.

18. Wang L, Wei J, Qian X, Yin H, Zhao Y, Yu L, Wang W, Liu B. ERCC1 and BRCA1 mRNA expression levels in metastatic malignant effusions is associated with chemosensitivity to cisplatin and/or docetaxel. BMC Cancer 2008:8:97.
19. Tiseo $M$, Bordi $P$, Bortesi B. ERCC 1/BRCA1 protein expressions and ERCC1/ XPD/XRCC1-3 gene polymorphisms as predictor factors of outcome in stage IIIB-IV nonsmall cell lung cancer (NSCLC) patients treated in first-line chemotherapy with or without cisplatin. Journal of Thoracic Oncology 2009;4:75-9.

20. Wachtersa FM, Wong LSM, Timensc W, Kampingab HH, Groena HJM ERCC1, hRad51, and BRCA1 protein expression in relation to tumour response and survival of stage III/IV NSCLC patients treated with chemotherapy. Lung Cancer 2005;50:211-9.

21. Kanga $\mathrm{CH}$, Jangb BG, Kimc DW, Chungb DH, Kima YT, Jheond $\mathrm{S}$, Sungd SW, Kima JH. The prognostic significance of ERCC1, BRCA1, XRCC1, and III-tubulin expression in patients with non-small cell lung cancer treated by platinum- and taxane-based neoadjuvant chemotherapy and surgical resection. Lung Cancer 2010;68(3):47883.

22. Deng CX. Roles of BRCA1 in DNA damage repair: a link between development and cancer. Human Molecular Genetics 2003;12:11-5.

23. Wang Y, Cortez D, Yazdi P, Neff N, Elledge SJ, Qin J. BASC, a super complex of BRCA1-associated proteins involved in the recognition and repair of aberrant DNA structures. Genes Development 2000;14:927-39.

24. MacLachlan TK, Somasundaram K, Sgagias $\bigotimes m$, Shifman $Y$, Muschel RJ, Cowan囚 KH, El-Deiry WS. BRCA1 effects on the cell cycle and the DNA damage response are linked to altered gene expression. Journal of Biological Chemistry 2000;275:2777-85.

25. Gowen LC, Avrutskaya AV, Latour AM, Koller BH, Leadon SA. BRCA1 required for transcription-coupled repair of oxidative DNA damage. Science 1998;281:1009-12.

26. Abbott DW, Thompson ME, Robinson-Benion C, Tomlinson $\mathrm{G}$, Jensen RA, Holt JT. BRCA1 expression restores radiation resistance in BRCA1-defective cancer cells through enhancement of transcription-coupled DNA repair. Journal of Biological Chemistry 1999;274:18808-12

27. Groch J. DNA-Repair Genes Predict Survival in Early Lung Cancer. Medpagetoday/Lung Cancer 2007. http://www.medpagetoday. com/HematologyOncology/LungCancer/5108

28. Albiges L, Andre F, Balleyguier C, Gomez-Abuin G, Chompret $A$,Delaloge $S$. Spectrum of breast cancer metastasis in BRCA1 mutation carriers: highly increased incidence of brain metastases Annals of Oncology 2005;16(11):1846-7. 